\title{
Fields at a Finite Conducting Wedge and Applications in Interconnect Modeling
}

\author{
Thomas Demeester and Daniël De Zutter, Fellow, IEEE
}

\begin{abstract}
The fields at a finite conducting 2-D wedge are studied by means of the surface admittance operator, and compared to the case of a perfect conductor. This technique, applied to a number of numerical examples, allows a thorough investigation of the singular behavior of the fields near the edge, including non-singular fields such as the longitudinal current distribution. Special attention is devoted to the validity of the quasi-TM approximations, when edge singularities are taken into account. The studied field properties lead to the formulation of an approximative local surface impedance for conductors, and are finally used to show how some differences in the resistive and inductive behavior of conductors with a different geometry are due to edge effects.
\end{abstract}

Index Terms-Wedge, edge effect, conductor, finite conductivity, skin effect, surface impedance, transmission line parameters, resistance, inductance.

\section{INTRODUCTION}

$\mathbf{F}$ OR many years, researchers have been looking for accurate descriptions of the loss mechanisms in interconnect structures. As modern technological applications in very-largescale integration (VLSI) circuits push the limits of speed and miniaturization, conductor losses more than ever remain an important issue, by far more relevant than radiation or dielectric losses. Not only heat generation needs to be kept under control, the losses also have an important impact on the signal integrity, due to attenuation and dispersion.

In a period of almost a hundred years, many authors have paid attention to the topic discussed in this paper, and in general to the skin effect and losses in rectangular conductors [1][19]. We thank the reviewer for bringing some of these papers to our attention.

The earliest research on interconnect losses is wellsummarized in [11], mentioning for instance Wheeler's incremental inductance rule, where the magnetic field generated by the axial current flow is used to calculate the losses, assuming an equal real and imaginary part of the highfrequency internal impedance per unit length. The resistive properties of coupled lines with finite conductivity were more rigorously studied in [16] and [17], using the Method of Moments (MoM) with a boundary discretization, respectively, a volume discretization of the field quantities. Many other numerical approaches were used for analogous purposes, e.g., Finite Elements Methods [12], hybrid methods based on the 'filament technique' at low frequencies and a surface integral equation at high frequencies [19], or a combination of the

The authors are with the Department of Information Technology, Ghent University, Sint-Pietersnieuwstraat 41, B-9000 Gent, Belgium. Tel: +32 9264 33 27, Fax: +329264 35 93. E-mail: thomas.demeester@intec.ugent.be, daniel.dezutter@ugent.be.
MoM and the Method of Lines [20], just to mention a few. Generally, the boundary integral equation techniques appear to be more suited in terms of both computation time efficiency and accuracy, than methods based on a volume discretization of the currents, especially at the highest frequencies.

The importance of the edge effect in the current profile of polygonal (mostly rectangular) conductors became clear with the (sometimes mutually inconsistent) results that were found from internal inductance calculations [21]-[23], and which clearly showed an important deviation from Wheeler's rule.

In parallel with the research on the effect of the finite conductivity on the circuit level properties (resistance, inductance) of the lines, another topic of investigation was the singular field behavior at edges. In [24], and further in [25], the cases of perfectly electric conducting (PEC) wedges and wedges with dielectric contrast were treated. A more detailed analysis and further references can be found in [26]. The theory of the singularity exponent as formulated in [25] was extended to finite conducting wedges in [27].

Although the specific field behavior at conductors' edges (both in the PEC and in the finite conducting case) and the current profile (relevant to the resistive and inductive properties of the lines) are intrinsically linked, both aspects were so far not examined simultaneously. On the one hand, 'circuit oriented' papers such as, e.g., [15], [18], [21], concentrate on the interconnect behavior, with no specific attention devoted to edge effects and their influence on the circuit parameters. On the other hand, [25] and [27] focus on the edge singularities only, not paying particular attention to the properties of the longitudinal field components, such as the current density, as these do not exhibit a singular behavior at the edges.

This paper describes the behavior of a finite conducting wedge, as a function of its opening angle $\alpha$, in combination with the longitudinal current profile. As opposed to the singularity exponent technique of [25] and [27], the applied method enables the description of the total edge field quantities, not restricted to the strongest singularity only. Although the technique is a numerical approximation obtained by the MoM, it is well-suited for an accurate description of the fields near an edge from low to very high frequencies, as it makes use of a boundary integral equation formulation, in combination with a field expansion that exactly describes the current crowding phenomenon inside the conductor.

Essential in this technique is the surface admittance matrix, which relates the electric field to the equivalent surface current densities that replace the conductors. The surface admittance matrix is calculated by means of a discretization of the Dirichlet to Neumann (DtN) operator of the considered conductor's cross-section. The method was first introduced for conductors 
in [28] and extended to dielectrics and semiconductors in [29]. In [30] and [31], the original method for rectangular conductors was extended to general polygonal shapes.

In Section II, a single wedge is considered. First, the relationship between Meixner's [25] work on field singularities and the quasi-TM approximations underlying the coupled transmission line model presented in [29] is elucidated. Next, the equivalent surface current density $j_{\text {eq }}$ as introduced in [28] and the current profile inside the wedge are studied. Subsequently, an approximative local surface impedance model for conductors is proposed, intended to demonstrate the principle physical properties of $j_{\mathrm{eq}}$. In a last subsection, the properties of the wedge current profile are used to reveal the influence of varying conductor angles on the per unit length (p.u.1.) resistance and inductance for conductors with a high but finite conductivity.

Finally, Section III summarizes the results.

\section{InVEStigation of the Edge EfFeCt}

This section is intended to provide the reader with some insight into the field distribution and essential phenomena that occur near edges. Focus is on the physics of the current and field distribution near a single edge, and the validity of the quasi-TM approximations (which is confirmed by numerical results).

The considered configuration consists of one triangular metallic non-magnetic conductor $S$ with conductivity $\sigma$, placed in free space. It is assumed that no other materials (dielectric or semiconducting substrates) are around, in order to study the edge effect in its most basic configuration, although the results are valid for more complicated structures as well. In order to avoid the proximity effect in the current distribution, the reference conductor is considered infinitely far away. All simulation results shown here are obtained by using the numerical method described in [30]. For the discretization of the boundary quantities, piecewise linear basis functions are used over a non-uniform grid. In this way a very fine grid can be used near the corner tips. When focussing on the edge effect only, the simulation frequency will be chosen sufficiently high, such that the influence of the side, opposite to the corner of interest, is negligible. In practice, this means the skin depth has to be much smaller than the distance between that corner and its opposite side.

\section{A. The Equivalent Surface Current Density}

Consider the triangle shown in Fig. 1 with an opening angle $\alpha$ at corner $T$, with the permeability $\mu_{0}$ of free space and a 'high' conductivity $\sigma$ (such that $\sigma \gg \omega \epsilon_{0}$ ). As explained above, we are only interested in the edge effect in the neighborhood of $T$. As mentioned in the Introduction, the volume current flowing through $S$, is replaced by an equivalent surface current density source $\mathbf{j}_{\mathrm{eq}}$ in free space on the boundary $c$ of $S$. This equivalent source is found from the requirement that outside $S$, it generates the original fields [28]

$$
\mathbf{j}_{\mathrm{eq}}=\mathbf{u}_{n} \times\left(\mathbf{h}^{+}-\mathbf{h}_{0}^{-}\right)=\mathbf{u}_{n} \times\left(\mathbf{h}^{-}-\mathbf{h}_{0}^{-}\right) .
$$

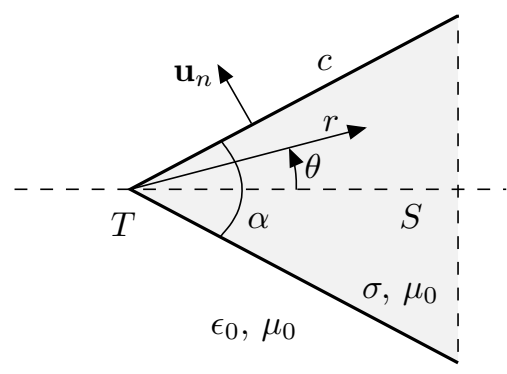

Fig. 1. Basic configuration of a conducting wedge (as part of a triangle), placed in free space.

with $\mathbf{u}_{n}$ the outward pointing normal unit vector on $c$. The superscripts + and - are used for the outer, respectively, the inner limit of the fields at $c$, and the subscript ' 0 ' indicates field quantities inside $S$ in the equivalent configuration, in which the material properties of $S$ are replaced by free space. To obtain the last part of (1), the continuity of the tangential magnetic field was invoked, which allows to write $\mathbf{j}_{\text {eq }}$ in terms of boundary quantities inside $S$ in the original, respectively, the equivalent configuration.

The quasi-TM approximations [29] dictate that for the determination of $e_{z}$ (to determine the resistive and inductive properties), the longitudinal component $j_{\mathrm{eq}, z}$ of the total equivalent current density suffices (as briefly motivated in the Appendix). For an $e^{-j \beta z+j \omega t}$-dependence of the fields, the transverse magnetic field $\mathbf{h}_{t}$ is found from Faraday's law

$$
j \omega \mu_{0} \mathbf{h}_{t}=j \beta \mathbf{u}_{z} \times \mathbf{e}_{t}-\nabla_{t} e_{z} \times \mathbf{u}_{z} .
$$

in which $\nabla_{t}=\partial / \partial x \mathbf{u}_{x}+\partial / \partial y \mathbf{u}_{y}$. Hence, $j_{\text {eq }, z}$, concisely written as $j_{\text {eq }}$, is given by

$$
j \omega \mu_{0} j_{\mathrm{eq}}=j \beta\left(e_{n}^{-}-e_{n 0}^{-}\right)+\frac{\partial e_{z}^{-}}{\partial n}-\frac{\partial e_{z 0}^{-}}{\partial n}
$$

with $e_{n}=\mathbf{e}_{t} \cdot \mathbf{u}_{n}$ and $\partial / \partial n$ the outward normal derivative.

In [25], Meixner presents an expansion of the fields near the edge, in order to investigate the field singularities. He shows that the longitudinal fields $e_{z}$ and $h_{z}$ do not display a singularity at the edge, and if there is no magnetic contrast (as is the case here), the total magnetic field remains finite. The $e_{z}$ field, e.g., can be expanded in polar coordinates $r$ and $\theta$ as

$$
e_{z}(r, \theta)=c_{1}(\theta) r^{\nu}+\ldots,
$$

omitting higher order terms in $r$, and with

$$
c_{1}(\theta)=-\frac{j \beta}{\nu} l \cos \nu \theta .
$$

with $l$ a complex integration constant. The transverse tangential and the normal electric field components exhibit a singular term that behaves as $r^{\nu-1}$. In [27], it is shown that in the case of a good conductor, $\nu$ can be well approximated by

$$
\nu=\frac{\pi}{2 \pi-\alpha} .
$$

which exactly dictates the singular behavior of $e_{n}$ in the case of a PEC conductor.

If the field expansions from [25] are inserted in (3), the singular terms in $j \beta e_{n}^{-}$and $\partial e_{z}^{-} / \partial n$ cancel each other out, as 
is also the case for $j \beta e_{n 0}^{-}$and $\partial e_{z 0}^{-} / \partial n$. Furthermore, these expansions allow to compare the singular terms in $\partial e_{z} / \partial n$ and $\partial e_{z 0} / \partial n$. Inside the wedge, $e_{z}$ satisfies

$$
\nabla_{t}^{2} e_{z}=\left(\beta^{2}-k^{2}\right) e_{z}
$$

with $k^{2}=-j \omega \mu_{0}(\sigma+j \omega \epsilon)$. The expansion (4) of $e_{z}$ is substituted in (7) and yields

$$
\left(\nu^{2} c_{1}(\theta)+\frac{\partial^{2} c_{1}(\theta)}{\partial \theta^{2}}\right) r^{\nu-2}+\ldots=\left(\beta^{2}-k^{2}\right) c_{1}(\theta) r^{\nu}+\ldots
$$

Setting the coefficient of $r^{\nu-2}$ to zero, confirms the $\theta$ behavior of $c_{1}(\theta)$ in (5), which is independent of the material parameters. The same remark holds for the term in $r^{\nu-1}$ in $\nabla_{t}^{2} e_{z}$. In the quasi-TM limit, the right-hand side of (7) becomes $j \omega \mu_{0} \sigma e_{z}$, and as seen from (8), this diffusion term is not relevant very close to the corner tip. A completely similar reasoning can be put forward for $e_{z 0}$ which satisfies

$$
\nabla_{t}^{2} e_{z 0}=\left(\beta^{2}-k_{0}^{2}\right) e_{z 0}
$$

with $k_{0}^{2}=\omega^{2} \epsilon_{0} \mu_{0}$. By construction, $e_{z 0}$ has the same boundary value on $c$ as $e_{z}$, and because replacing $k^{2}$ by $k_{0}^{2}$ in (7) has no influence on the singular behavior, the two highest order terms of the expansions of $e_{z}$ and $e_{z 0}$ are identical. As a consequence, the singularity in $\partial\left(e_{z}-e_{z 0}\right) / \partial n$ is cancelled out, as well as the first higher order term $\left(\propto r^{\nu}\right)$.

The above reasoning shows that all four field components in the right-hand side of (3) contain the same singular term. Leaving these singular terms out, thus has no influence on $j_{\text {eq }}$. Let us indicate the fields in (3) without their singular term with the caret symbol "^". Within the quasi-TM limit, the terms $j \beta \hat{e}_{n}^{-}$and $j \beta \hat{e}_{n 0}^{-}$are both negligible with respect to $\partial \hat{e}_{z}^{-} / \partial n$ (considering the fact that $\left|\partial \hat{e}_{z}^{-} / \partial n\right|>\left|\partial \hat{e}_{z 0}^{-} / \partial n\right|$ due to the current crowding effect). The reason for this is twofold. On the one hand, the longitudinal wavelength is much larger than a typical cross-sectional distance over which the fields extend, and therefore we can in general say that " $j \beta \ll \partial / \partial n$ " for a certain field quantity. On the other hand, $\left|\hat{e}_{n}^{-}\right| \ll\left|\hat{e}_{z}^{-}\right|$. Taking these two arguments into account leads to

$$
\begin{aligned}
j_{\mathrm{eq}} & =\frac{1}{j \omega \mu_{0}}\left(\frac{\partial \hat{e}_{z}^{-}}{\partial n}-\frac{\partial \hat{e}_{z 0}^{-}}{\partial n}\right) \\
& =\frac{1}{j \omega \mu_{0}}\left(\frac{\partial e_{z}^{-}}{\partial n}-\frac{\partial e_{z 0}^{-}}{\partial n}\right) .
\end{aligned}
$$

This proves that in the quasi-TM approximation expression (11), already put forward in [28] for the $z$-independent TM case, still remains valid when the singular field behavior at an edge is accounted for. As not only the singular terms in $r^{\nu-1}$ in (11) cancel out, but also the nonsingular terms in $r^{\nu}$ and a possible constant term, $j_{\text {eq }}$ will become zero at the tip $T$, as will be confirmed by the numerical examples.

In the sequel, $j_{\mathrm{eq}}$ for a finite conductor will be compared to the surface current density $j_{\mathrm{PEC}}$ on a PEC wedge. This is motivated by the following observation. As the fields inside the PEC conductor are zero, the inside can be substituted by free space, provided proper surface charges and surface currents are placed on the boundary. If these sources are equal to the original surface charge and current on the PEC conductor, the

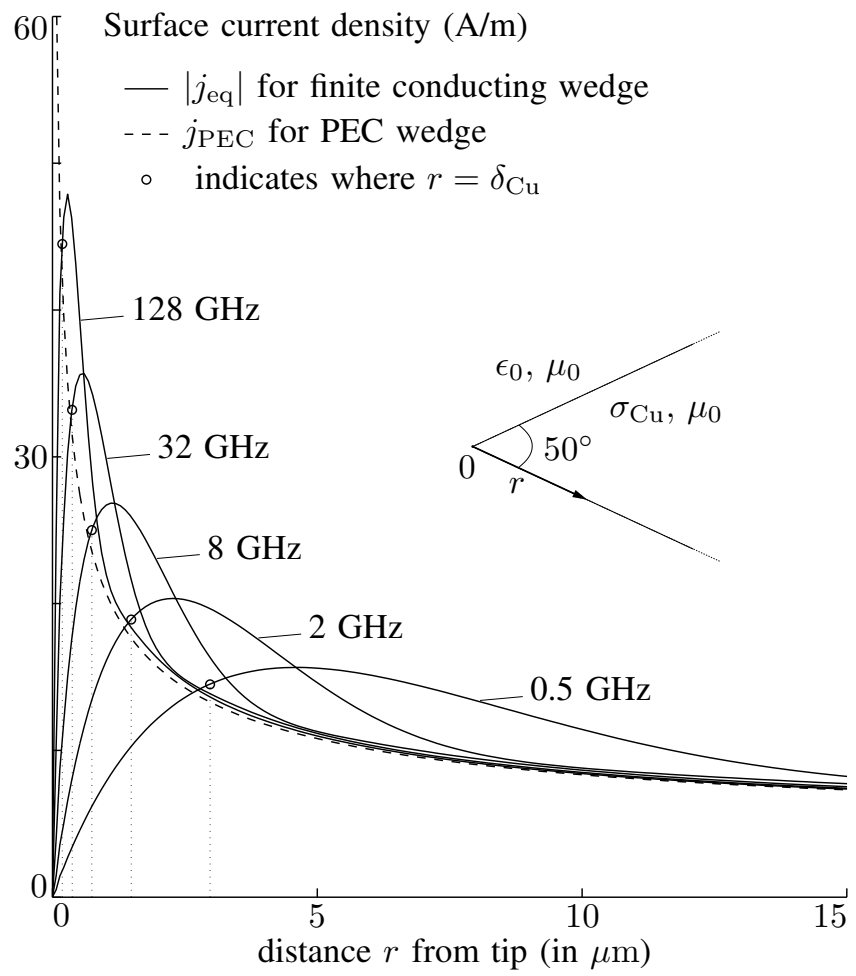

Fig. 2. Surface current density $\left|j_{\text {eq }}\right|$ for a $50^{\circ}$ copper wedge (see inset). Solid lines: $\left|j_{\text {eq }}\right|$ for finite conductivity $\left(\sigma_{\mathrm{Cu}}=57.2 \mathrm{MS} / \mathrm{m}\right)$, with indication of the distance $\delta_{\mathrm{Cu}}$ from the corner top. Dashed line: $\left|j_{\mathrm{PEC}}\right|$ for PEC wedge.

fields in both configurations are the same and the boundary conditions are met. $j_{\mathrm{PEC}}$ is hence the 'equivalent current source' for the PEC problem, and is in this paper compared to the finite conducting case. $j_{\mathrm{PEC}}$ can be obtained by solving a static potential problem, with the longitudinal magnetic vector potential $a_{z}=V \sqrt{\epsilon_{0} \mu_{0}}$ on $c$. This result is obtained from the general relationship

$$
e_{z}=j \beta \phi-j \omega a_{z},
$$

combined with $e_{z}=0, \phi=V$ and $\beta=\omega \sqrt{\epsilon_{0} \mu_{0}}$ on the boundary of the considered perfect conductor in free space.

In Fig. 2, the equivalent surface current density $\left|j_{\text {eq }}\right|$ near a copper wedge of $50^{\circ}$ is compared with the PEC case, for various frequencies. In the simulation, the wedge was the top corner of an equilateral triangle (with both legs $60 \mu \mathrm{m}$ long, such that the edge effects of the different corners do not interfere at the shown frequencies), with the electric boundary potential put to $V=1 \mathrm{~V}$. To get an idea of the frequency relative to the dimensions, the point where the distance $r$ to the corner tip equals one skindepth $\delta_{\mathrm{Cu}}$, is indicated as well. Notice that, as explained earlier, $j_{\text {eq }}$ vanishes at the tip and therefore does not have $j_{\mathrm{PEC}}$ as its limiting case for $\omega \sigma \rightarrow \infty$, because $j_{\mathrm{PEC}}$ is always singular (for an wedge angle smaller than $180^{\circ}$ ). It is clear though, that more than a few skindepths away from the edge, $j_{\mathrm{eq}}$ and $j_{\mathrm{PEC}}$ become almost identical.

In Fig. 3, a similar wedge is treated as in Fig. 2, but at a fixed frequency $(10 \mathrm{GHz})$ and for a varying top angle $\alpha$. In order to investigate the behavior of $j_{\text {eq }}$ with respect to $j_{\text {PEC }}$ for 


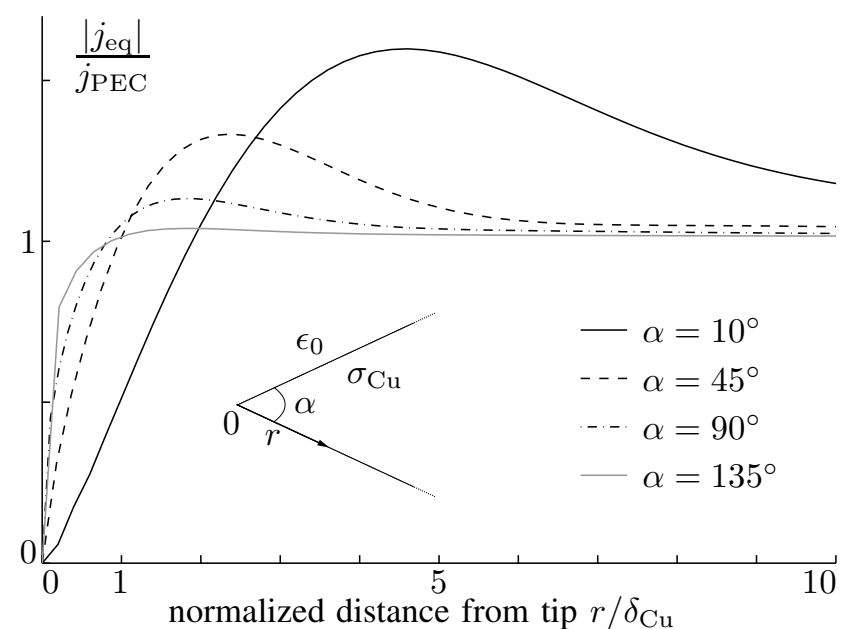

(a)

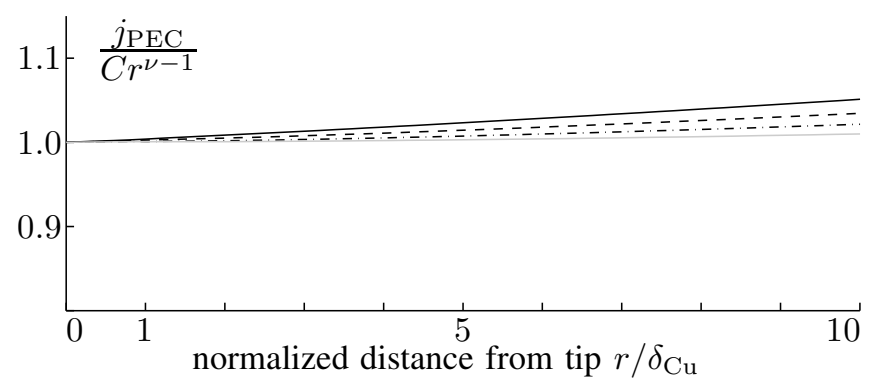

(b)

Fig. 3. Normalized surface current densities for different angles $\alpha$, and at $10 \mathrm{GHz}$. (a) $\left|j_{\mathrm{eq}}\right| / j_{\mathrm{PEC}}$, and (b) $j_{\mathrm{PEC}} / C r^{\nu-1}$, with $C$ chosen such, that $j_{\mathrm{PEC}} / \mathrm{Cr} r^{\nu-1}$ becomes one at $r=0$.

different values of $\alpha$, Fig. 3 (a) shows the normalized current density $\left|j_{\text {eq }}\right| / j_{\text {PEC }}$ near the corner tip. As the solution of the diffusion equation only depends on the product $\omega \sigma$ instead of both factors separately, the abscis $r$ is normalized by the skin depth in copper $\delta_{\mathrm{Cu}}=\left(\pi f \mu_{0} \sigma_{\mathrm{Cu}}\right)^{-1 / 2}$. The deviation of the curves in Fig. 3 (a) from unity shows the influence of the finite conductivity. The equivalent current density at the sharpest angles deviates more from $j_{\mathrm{PEC}}$ than at the wider angles. This can be explained by the diffusion at skin effect frequencies. Near the edge of a narrow wedge, the adjacent sides are more tightly coupled for a wider wedge, and the current crowding effect starts appearing further away from the tip as compared to the wide wedge case. Therefore, the edge effect is more important for narrower wedges. In [30], this phenomenon appears to be the reason for the slower convergence of the ICWM (Iterative Combined Waveguide Modes) procedure for sharper angles, where the coupling between the sides of the conductor is gradually taken into account.

As a verification of the singular behavior of $j_{\mathrm{PEC}}$, Fig. 3 (b) shows $j_{\mathrm{PEC}}$ for the same wedge, normalized by a factor $\mathrm{Cr}^{\nu-1}$, with $\nu$ given by (6). For $\pi>\alpha>0$, the singularity exponent $(\nu-1)$ lays between 0 and $-1 / 2$, with, e.g., an $r^{-1 / 3}$-behavior for a $90^{\circ}$ angle. For each value of $\alpha$, the proportionality constant $C$ is chosen such that $\left(j_{\mathrm{PEC}} / \mathrm{Cr}^{\nu-1}\right)$ becomes one in the limit for $r=0$.

\section{B. The Electric Boundary Potential $\phi_{c}$}

This paragraph is intended to demonstrate the validity of the assumption that, in the quasi-TM limit, the electric potential $\phi$ on the boundary of a good conductor remains constant over its edges. With Meixner, the potential $\phi$ can be written as

$$
\phi(r, \theta)=V+f_{1}(\theta) r^{\nu}+f_{2}(\theta) r^{\nu+1}+\ldots
$$

The expansions in [25] only describe the singular behavior of the fields (or their normal derivatives) and here, (13) is completed with a constant term $V$, as motivated in [32]. The coefficient $f_{1}(\theta)$ is found as

$$
f_{1}(\theta)=-\frac{l \cos \nu \theta}{\nu}
$$

and the term $f_{1}(\theta) r^{\nu}$ has a singular normal derivative at $r=0$. From (5) and (12), we see that neglecting this term with respect to the total potential $\phi$, corresponds to neglecting the term $c_{1}(\theta) r^{\nu}$ in $e_{z}$. This means that an excitation with a constant boundary value $\phi_{c}=V$, does not give rise to a singularity in $\partial e_{z} / \partial n$. This approximation is acceptable within the quasi-TM limit, as is briefly discussed in the Appendix.

We will demonstrate with a numerical example how accurate this approximation really is, by comparing the approximative constant voltage excitation $\phi_{c}=V$ with the term $f_{1}(\theta) r^{\nu}=\Delta \phi_{c}$ on the boundary $\theta=\alpha / 2$ of a wedge (see Fig. 1). Near the edge, where the singular term proportional to $r^{\nu-1}$ dominates the surface charge $\rho_{\text {eq }}$ (see Appendix), $e_{n}^{-}$ is, with the results from [25], given by

$$
e_{n}^{-} \approx-l \sin \frac{\nu \alpha}{2} r^{\nu-1} \approx \frac{j \omega}{\sigma} \rho_{\mathrm{eq}}
$$

such that

$$
\Delta \phi_{c} \approx \frac{j \omega}{\sigma} \frac{\rho_{\mathrm{eq}}}{\nu r^{\nu-1}} \cot \left(\frac{\nu \alpha}{2}\right) r^{\nu} .
$$

Note that $\rho_{\mathrm{eq}}$ is a very good approximation of the actual surface charge, even though calculated with the approximative excitation $\phi_{c}=V$, as it is very similar to the PEC case where $\phi_{c} \equiv V$ (see Appendix). Note that $\rho_{\text {eq }}$ itself is proportional to $r^{\nu-1}$ and hence that $\Delta \phi_{c}$ tends to zero as $r^{\nu}$.

For the copper $50^{\circ}$ wedge from Fig. 2 and at the same frequencies, the term $\Delta \phi_{c}$ is compared with the voltage $\phi_{c}=V$, the constant excitation voltage used in the quasiTM simulations. Fig. 4 shows the results, and it appears that even at the highest shown frequencies, $\Delta \phi_{c}$ remains many orders of magnitude smaller than $\phi_{c}$, for the shown region $r \in[0,15 \mu \mathrm{m}]$ where the approximation (16) can be assumed to be valid.

\section{The Electric Field Distribution Inside the Wedge}

The longitudinal electric field $e_{z}$ on a conductor's boundary can be found with the MoM [29], and the inside $e_{z}$ field is readily determined as well, by means of an expansion in terms of parallel plate waveguide modes as described in [30]. In Fig. 5, the normalized longitudinal electric field distribution $\left|e_{z} / j \beta \phi_{c}\right|$ is shown inside the $50^{\circ}$ copper wedge of Fig. 2 and for a radial length of $r=15 \mu \mathrm{m}$, at $10 \mathrm{MHz}, 100 \mathrm{MHz}$, $1 \mathrm{GHz}$, and $10 \mathrm{GHz}$. For these frequencies, the skin depth is given, respectively, by $21 \mu \mathrm{m}, 6.6 \mu \mathrm{m}, 2.1 \mu \mathrm{m}$ and $0.66 \mu \mathrm{m}$. 


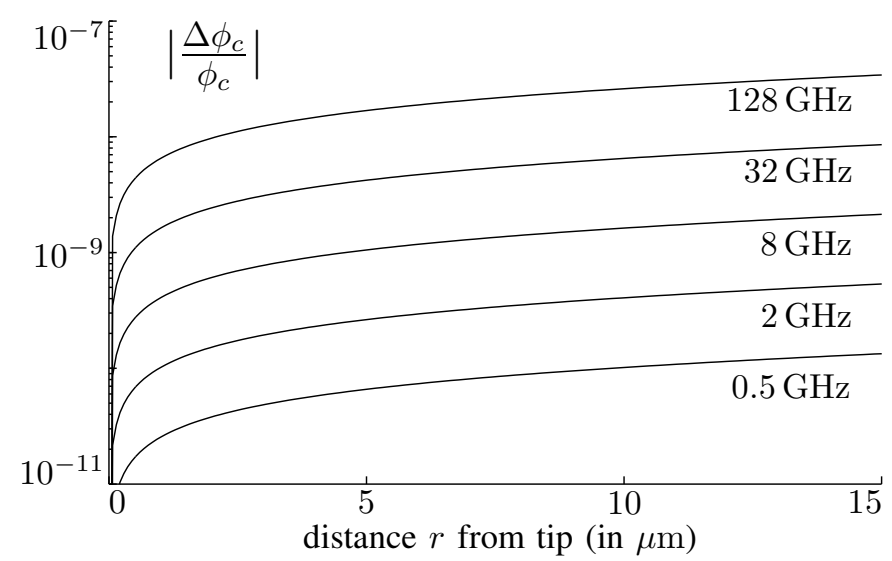

Fig. 4. The term $\Delta \phi_{c}$ in the boundary potential expansion near the edge, in absolute value and normalized by $\phi_{c}$, for the $50^{\circ}$ wedge shown in Fig. 2
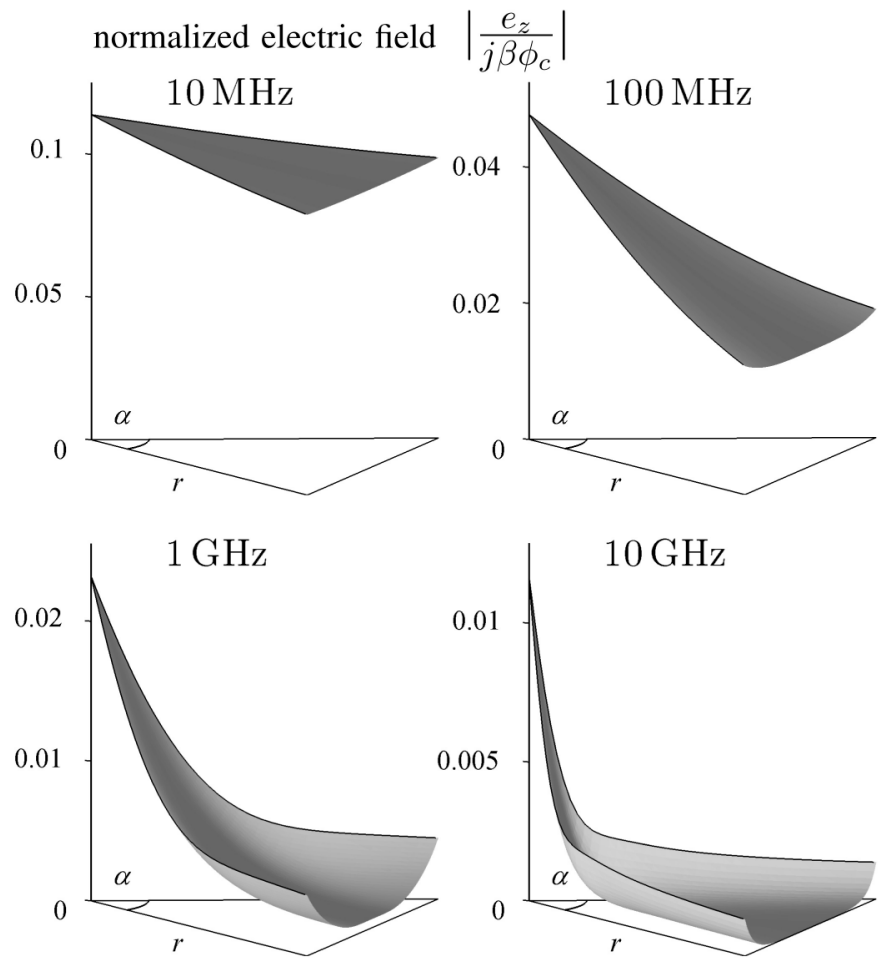

Fig. 5. Normalized electric field $\left|e_{z} / j \beta \phi_{c}\right|$ inside a copper wedge $\left(\sigma_{\mathrm{Cu}}=\right.$ $57.2 \mathrm{MS} / \mathrm{m}$ ) with an angle $\alpha=50^{\circ}$, shown for $r \in[0,15 \mu \mathrm{m}]$.

In order to visualize the influence of the wedge angle on the current distribution, Fig. 6 (a) again shows the normalized electric field $\left|e_{z} / j \beta \phi_{c}\right|$, and Fig. 6 (b) shows the phase of $e_{z}$ on $c$. The same geometries are used as for the simulations shown in Fig. 3. The increase of $e_{z}$ toward the edge is much more pronounced for the sharpest angles, and almost nonexisting for the obtuse angle of $135^{\circ}$. The phase $\arg \left(e_{z}\right)$ for the sharp corners displays a large deviation of the plane wave limit of $45^{\circ}$, over a distance of many skindepths away from the tip.

As a verification of the numerical results, the boundary
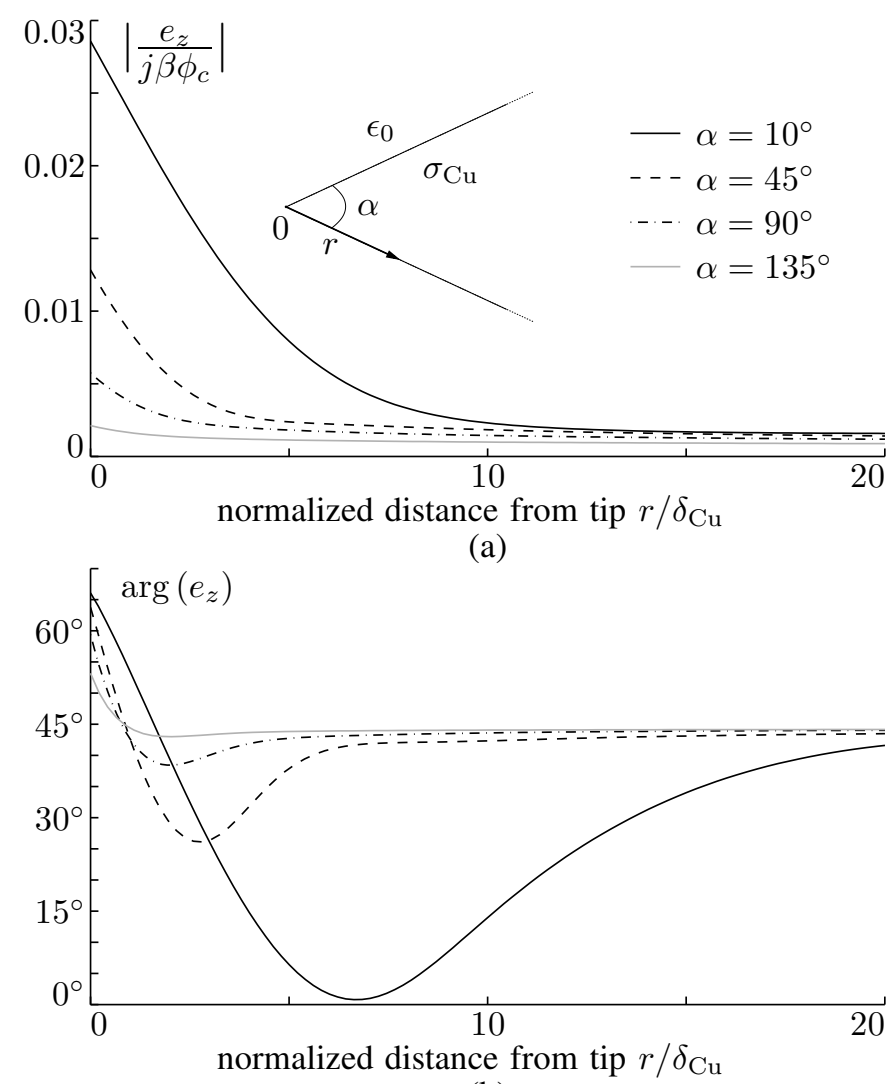

(b)

Fig. 6. (a) The normalized electric field $\left|e_{z} / j \beta \phi_{c}\right|$ on the boundary of a copper wedge $\left(\sigma_{\mathrm{Cu}}=57.2 \mathrm{MS} / \mathrm{m}\right)$ for different angles $\alpha$ and at $10 \mathrm{GHz}$, and (b) the phase of the corresponding electric field $e_{z}\left(\right.$ for $\left.\phi_{c}=1 \mathrm{~V}\right)$.

value of $e_{z}$ on a rectangular conductor is compared with results found in [17]. The simulated configuration consists of a golden microstrip line above a ground plane. Fig. 7 shows the geometry (see inset), as well as the results calculated by means of the MoM in combination with the DtN operator in solid lines, at different frequencies. At the frequency $f_{0}$, the reference data from [17] are indicated as well. Note that in [17], a golden ground plane was used, whereas here just a PEC ground is considered. Yet the results seem to match quite accurately. In [17], the fields were considered to be $z$-independent, and excited by means of the external field $E_{z}^{\mathrm{ext}}$, which corresponds with our term $j \beta \phi_{c}$ in (12). In Fig. 7, the normalized field $\left|e_{z} / j \beta \phi_{c}\right|$ is given. At the lowest frequencies, $j \omega a_{z} \ll e_{z}$, such that the current distribution is almost uniform and $e_{z} / j \beta \phi_{c} \approx 1$. At skin effect frequencies, $e_{z}$ decreases because the difference between $j \beta \phi$ and $j \omega a_{z}$ goes to zero for $\omega \sigma \rightarrow \infty$, as the fields approach the PEC field distribution with $e_{z} \equiv 0$.

\section{A Local Surface Impedance Approximation}

After studying $j_{\text {eq }}$ and $e_{z}$ near a wedge, we have a sufficient understanding on the wedge effects as to propose the following approximation for $j_{\text {eq }}$ on the boundary $c$ of an arbitrary twodimensional conductor in free space (hence not restricted to 


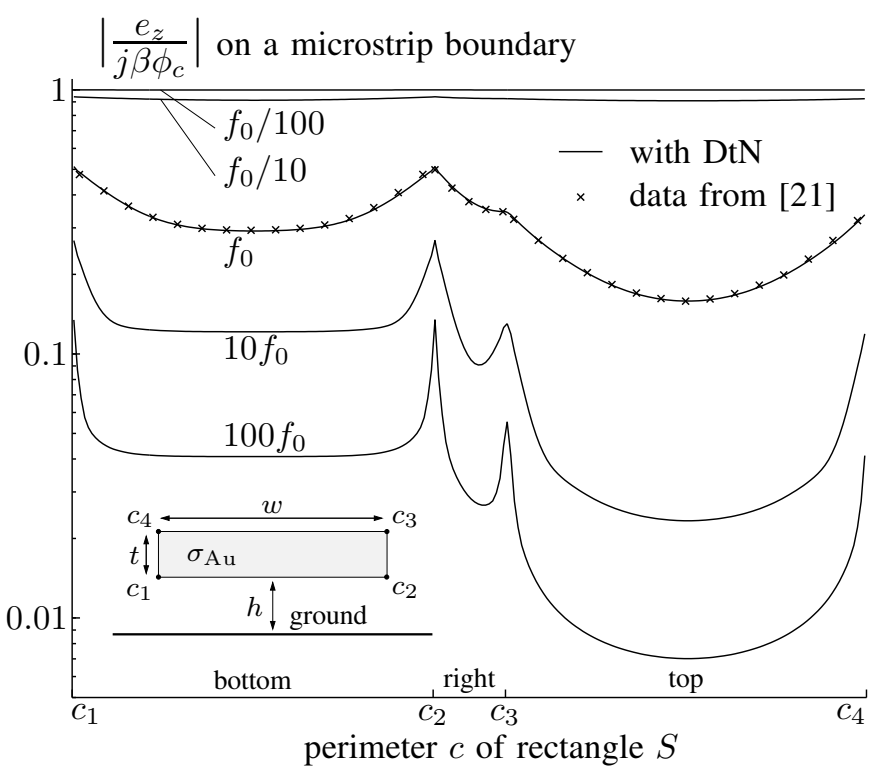

Fig. 7. Normalized longitudinal electric field $\left|e_{z} / j \beta\right|$ on the boundary of the microstrip, shown in the inset, with $w=10 \mu \mathrm{m}, t=2 \mu \mathrm{m}, h=2 \mu \mathrm{m}$, and $\sigma_{\mathrm{Au}}=41 \mathrm{MS} / \mathrm{m}$. The simulations were performed for different frequencies, with $f_{0}=4.367 \mathrm{GHz}$.

the wedge only)

$$
j_{\mathrm{eq}} \approx \frac{1}{j \omega \mu_{0}}\left(e_{z} \frac{\partial e_{1}}{\partial n}\right)_{c} \stackrel{\text { def }}{=} j_{\text {approx }} .
$$

The approximate surface current density $j_{\text {approx }}$ contains the actual boundary value of $e_{z}$, multiplied with the normal derivative of a function $e_{1}$ which satisfies

$$
\nabla_{t}^{2} e_{1}=j \omega \mu_{0} \sigma e_{1}
$$

inside the conductor (as $e_{z}$ itself), but is 1 on $c$. The idea behind this approximation is the separation of the two major phenomena that play a role. On the one hand, we have the value of $e_{z}$ on $c$, increasing towards the corners as governed by the (outside) magnetic field, and on the other hand the current crowding phenomenon inside the conductor, which would behave like $\partial e_{1} / \partial n$ if the boundary field were a constant. At the higher frequencies and far enough away from the corners, the local plane wave behavior confirms that the approximation becomes accurate. At the lower frequencies, $e_{z}$ is approximately constant over the cross-section, and the approximation holds as well. With (17), we have therefore constructed a local surface impedance $Z_{\text {approx }}$,

$$
Z_{\text {approx }}=\frac{j \omega \mu_{0}}{\frac{\partial e_{1}}{\partial n}}
$$

with the correct low- and high-frequency limits. The approximation is acceptable, because the total current $I$ is, for any frequency, found as

$$
I=\oint_{c} j_{\mathrm{eq}} d c=\oint_{c} j_{\text {approx }} d c .
$$

This can be proved by invoking Green's theorem in combination with (17), (18) and the same diffusion equation for $e_{z}$.
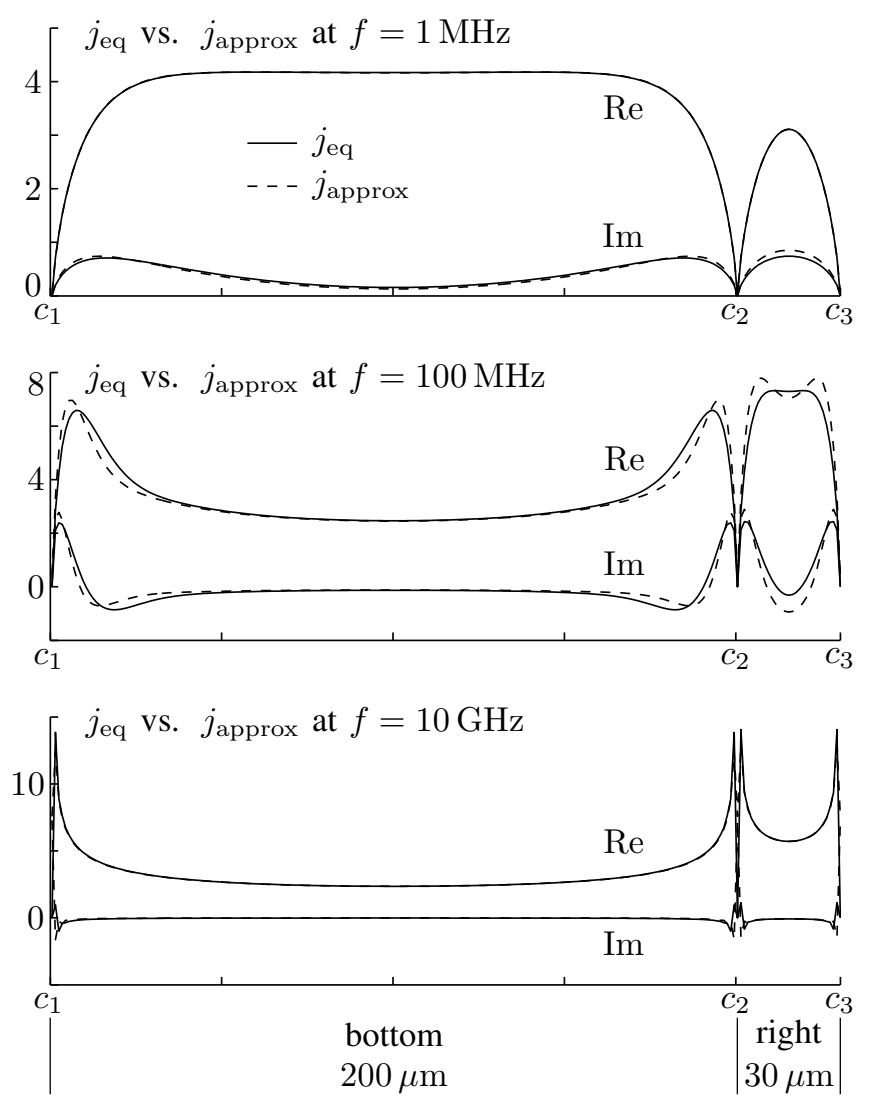

Fig. 8. Real and imaginary part of $j_{\text {eq }}$ and $j_{\text {approx }}$, at (a) $1 \mathrm{MHz}$, (b) $100 \mathrm{MHz}$, and (c) $10 \mathrm{GHz}$ for a rectangular copper conductor in free space $\left(\sigma_{\mathrm{Cu}}=58 \mathrm{MS} / \mathrm{m}\right.$, width $=200 \mu \mathrm{m}$, height $\left.=30 \mu \mathrm{m}\right)$.

As a numerical verification, consider a rectangular copper conductor $\left(\sigma_{\mathrm{Cu}}=58 \mathrm{MS} / \mathrm{m}\right)$ with dimensions $200 \mu \mathrm{m} \times$ $30 \mu \mathrm{m}$ in free space. At $1 \mathrm{MHz}, 100 \mathrm{MHz}$, and $10 \mathrm{GHz}$ ), the real and imaginary part of both $j_{\text {eq }}$ and $j_{\text {approx }}$ are shown in Fig. 8. For these frequencies, the skindepth in copper is, respectively, $66 \mu \mathrm{m}, 6.6 \mu \mathrm{m}$, and $0.66 \mu \mathrm{m}$. At the low and high frequencies, $j_{\text {eq }}$ and $j_{\text {approx }}$ are very close to one another as expected, but also at the intermediate frequency the behavior is quite similar, confirming the physical ideas behind the equivalent surface current density.

\section{E. Influence of the Edge Effect on the p.u.l. Resistance and Inductance}

In [30], it was found that the high-frequency resistance for trapezoidal or triangular conductors is higher than for a rectangular reference conductor with the same cross-sectional area, despite their longer circumference. In a last numerical example, the field distribution for these situations is shown in direct relationship with the corresponding circuit properties.

The starting point is the following telegrapher equation for a single line

$$
j \beta V=(j \omega L+R) I=Z I .
$$

Studying $e_{z} / j \beta V$ on the conductor's cross-section, or its normal derivative at the boundary, only yields information on 


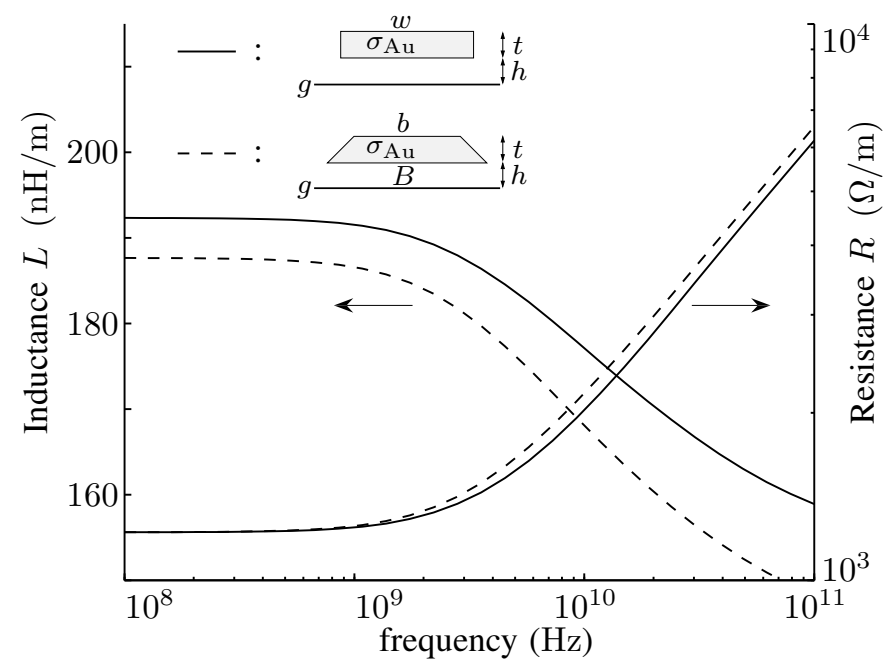

Fig. 9. Resistance and Inductance p.u.1. for a golden conductor (solid lines: rectangular; dashed lines: trapezoidal) placed a distance $h=2 \mu \mathrm{m}$ above a PEC ground plane $g$. The dimensions are $w=10 \mu \mathrm{m}, t=2 \mu \mathrm{m}, B=$ $12 \mu \mathrm{m}$, and $b=8 \mu \mathrm{m}$.

$Z^{-1}$, not directly on the resistance and inductance of the line. Therefore, we will transform (21) into

$$
(j \omega L+R)=j \beta \tilde{V} \oint_{c}\left(\frac{1}{j \omega \mu_{0}} \frac{\partial e_{z}^{-}}{\partial n}\right)^{*} d c .
$$

with the excitation voltage $\tilde{V}$ chosen real and positive, but such that $|I|=1 \mathrm{~A}$ (or, alternatively, $\tilde{V}=V /|I|^{2}$ ). The integrand equals the complex conjugate $h_{\mathrm{tan}}^{*}$ of the tangential magnetic field in the quasi-TM limit. The contribution of $\partial e_{z 0} / \partial n$ could be included as well, to obtain $j_{\mathrm{eq}}$ in the integrand (as it does not contribute to the integration), but this would make the graphical results less transparant.

The first considered configuration consists of a rectangular golden conductor with width $w=10 \mu \mathrm{m}$ and height $t=$ $2 \mu \mathrm{m}$, placed above a PEC ground plane with a separation of $h=2 \mu \mathrm{m}$. This is the configuration used in Fig. 7, and operated at $10 \mathrm{GHz}$. Secondly, a symmetric trapezoidal golden conductor is considered, also placed above a PEC ground plane, with a bottom width $B=12 \mu \mathrm{m}$ and a top width $b=8 \mu \mathrm{m}$, and hence the same area as its rectangular counterpart.

Fig. 9 shows the resistance $R$ and the inductance $L$ of both configurations. The inductance of the trapezoidal conductor is considerably lower than the rectangle's, and the resistance displays the opposite behavior.

According to (22), the circuit parameters $\omega L$ and $R$ are obtained by integration of the quantities $\operatorname{Im}\left(j \beta \tilde{V} h_{\mathrm{tan}}^{*}\right)$ and $\operatorname{Re}\left(j \beta \tilde{V} h_{\tan }^{*}\right)$ shown in, respectively, Fig. 10 (a) and (b). The main difference in $j \beta \tilde{V} h_{\mathrm{tan}}^{*}$ between bottom and top side is governed by the proximity effect. There is a very close resemblance on the top side of both conductors, with a considerably lower tangential magnetic field than on the bottom side. It means that the $135^{\circ}$ angles of the trapezoidal conductor have very little influence on the field distribution, confirming previous investigations at wedges. For the bottom

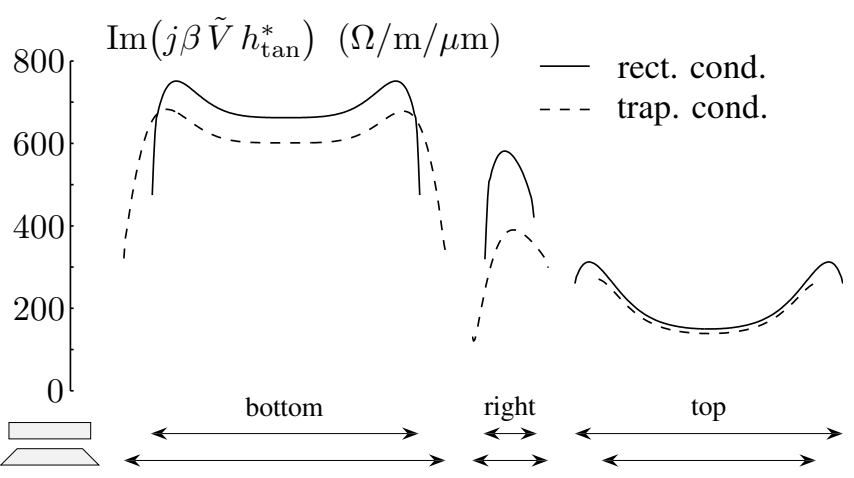

(a)

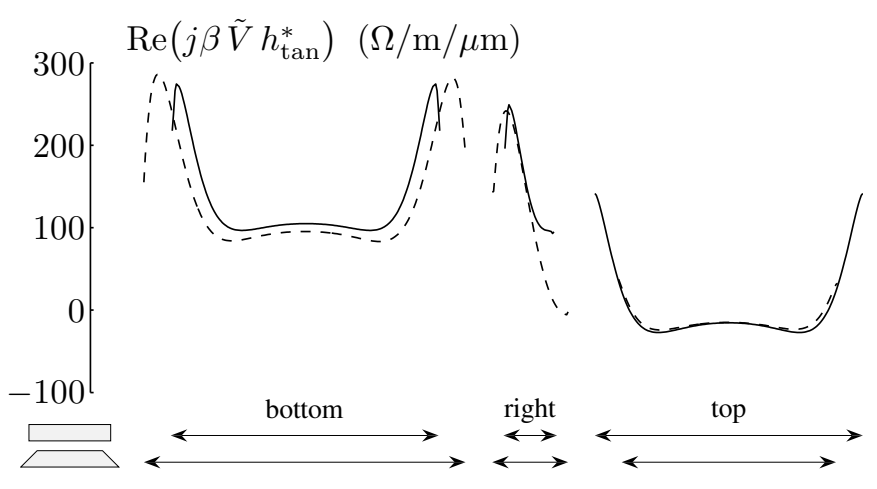

(b)

Fig. 10. Comparison of $j \beta \tilde{V} h_{\mathrm{tan}}^{*}$ on the boundary of the rectangular vs. the trapezoidal conductor (each above a PEC ground plane), used in Fig. 9.

sides, there is a larger deviation, primarily due to the proximity effect as well. As the bottom side of the trapezoidal conductor is larger than the corresponding side for the rectangle, and given the fact that the total current flowing through both conductors remains fixed at $1 \mathrm{~A}$ and is primarily located in the lower part of the conductor, due to the proximity effect, the agerage bottom field away from the corners is smaller in the trapezoidal case as compared to the rectangular one. This translates into a lower $L$-value. The authors have verified that the current density distribution shows a similar behavior. However, near the sharp $45^{\circ}$ angles, the phase shift in the current density (see also Fig. 6 (b)) and therefore also in the tangential magnetic field, has the effect that the resistive term $\operatorname{Re}\left(j \beta \tilde{V} h_{\tan }^{*}\right)$ is no longer smaller than at the $90^{\circ}$ angles of the rectangle, but even slightly higher, resulting in an overall higher p.u.l. resistance $R$ at skin effect frequencies.

\section{CONCLUSION}

The quasi-TM techniques for modeling the resistive and inductive properties of conductors remain valid near conductor edges. The specific field behavior of a finite conducting wedge was investigated and illustrated with a number of numerical examples to clarify the underlying physical mechanisms, which led to the formulation of an approximative local surface impedance description of conductors. Finally, the studied phenomena were used to explain some differences in the $(R, L)$ behavior of microstrips with different geometries. 


\section{APPENDIX}

This Appendix deals with some issues, related to the quasiTM approximations. In the frequency range where they are valid, $\left|j_{\text {eq, } z}\right| \gg\left|j_{\text {eq, tan }}\right|$. The reason is, with (1), that

$$
\left|h_{\tan }\right| \gg\left|h_{z}\right|
$$

(with the subscript tan denoting the transverse part of the total tangential field component), as $h_{\text {tan }}$ has a static contribution, whereas $h_{z}$ is only of second order in $\omega$. However, to keep the quasi-TM equations consistent, $j_{\text {eq, tan }}$ cannot be considered zero, as it is relevant for the capacitive behavior. Indeed, the surface charge $\rho_{\text {eq }}$, associated with the total equivalent current density is found as

$$
-j \omega \rho_{\mathrm{eq}}=\frac{\partial j_{\mathrm{eq}, \mathrm{tan}}}{\partial \tan }-j \beta j_{\mathrm{eq}, z} .
$$

The surface charge, associated with the normal electric field, is singular at the edges, as is $\partial j_{\text {eq, } \tan } / \partial \tan$, while $j \beta j_{\text {eq, } z}$ remains finite. Moreover, the variation of $j_{\mathrm{eq}, z}$ in the $z$ direction is small, compared to variations in the cross-section. Therefore, the term in $j_{\text {eq, tan }}$ is essential in (24). In practice, the influence of $j_{\text {eq,tan }}$ is well taken care of, if a surface charge source $\rho_{\mathrm{eq}}$ is directly imposed on the surface, together

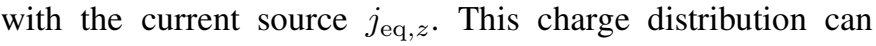
be determined by solving a quasi-static potential problem replacing the actual conductor with a PEC one, as motivated in [29]. This approach remains valid at the edges as well, because the singular term in $\rho_{\text {eq }}$ has an $r^{\nu-1}$ behavior, and for a good conductor, $\nu$ approximately equals its value for a PEC conductor [27].

As shown earlier, the approximation $\phi_{c}=V$ corresponds to omitting the term $c_{1}(\theta) r^{\nu}$ in $e_{z}$. That (finite) term keeps up for the 'slow' longitudinal variation of the (singular) transverse current, because the curl of the total electric field has to remain finite. The field $h_{z}$ is associated with the transverse electric field (more specifically, its transverse rotation), and similarly, the much larger $h_{\tan }$ with the part of $e_{z}$ without the term $c_{1}(\theta) r^{\nu}$ (by means of its normal derivative). Within the quasi-TM limit (23), we can therefore say that near the edge $\left|e_{z}\right| \gg\left|c_{1}(\theta) r^{\nu}\right|$ and hence the approximation $\phi_{c}=V$ is sufficiently accurate (confirmed also in Fig. 3).

\section{REFERENCES}

[1] A. Press., "Resistance and reactance of massed rectangular conductors," Phys. Rev., vol. 8, no. 4, pp. 417-422, Oct. 1916.

[2] H. Schwenkhagen, "Untersuchungen über Stromverdrängung in rechteckigen Leitern," Archiv für Elektrotechnik, vol. 17, no. 6, pp. 537589, 1927.

[3] D. Cockcroft, J., "Skin effect in rectangular conductors at high frequencies," Proc. Roy. Soc., vol. 122, no. 790, pp. 533-542, Feb. 1929.

[4] S. J. Haefner, "Alternating-current resistance of rectangular conductors," Proc. IRE, vol. 25, pp. 434-447, Apr. 1937.

[5] H. G. Groß, "Die Berechnung der Stromverteilung in zylindrischen Leitern mit rechteckigem und elliptischem Querschnitt," Archiv für Elektrotechnik, vol. 34, no. 5, pp. 241-268, May 1940.

[6] F. Lettowsky, "Eine Methode zur Berechnung des Hochfrequenzwiderstandes zylindrischer Leiter allgemeiner Querschnittsform," Archiv für Elektrotechnik, vol. 41, no. 1, pp. 64-72, Jan. 1953.

[7] P. Silvester, "Modal network theory of skin effect in flat conductors," Proc. IEEE, vol. 54, no. 9, pp. 1147-1151, Sep. 1966.

[8] - "The accurate calculation of skin effect in conductors of complicated shape," IEEE Trans. Power App. Syst., vol. PAS-87, no. 3, pp. 735-742, Mar. 1968.
[9] L. Pouplier, "Berechnung des komplexen Wechselstromwiderstandes von zylindrischen Leitern mit rechteckigem Querschnitt," ETZ-A, vol. 89, no. 22, pp. 611-617, 1968.

[10] P. Hammond and J. Penman, "Calculation of eddy currents by dual energy methods," Proc. Inst. Elec. Eng. IEE, vol. 125, pp. 701-708, 1978.

[11] E. Denlinger, "Losses of microstrip lines," IEEE Trans. Microw. Theory Tech., vol. 28, no. 6, pp. 513-522, Jun 1980.

[12] L. Olson, "Application of the finite element method to determine the electrical resistance, inductance, capacitance parameters for the circuit package environment," IEEE Trans. Compon., Hybrids, Manuf. Technol., vol. 5, no. 4, pp. 486-492, Dec. 1982.

[13] M. Krakowski and H. Morawska, "Skin effect and eddy currents in a thin tape," Archiv für Elektrotechnik, vol. 66, no. 2, pp. 95-98, Mar. 1983.

[14] P. Waldow and I. Wolff, "The skin-effect at high frequencies," IEEE Trans. Microw. Theory Tech., vol. 33, no. 10, pp. 1076 - 1082, Oct. 1985.

[15] A. Djordjevic, T. Sarkar, and S. Rao, "Analysis of finite conductivity cylindrical conductors excited by axially-independent tm electromagnetic field," IEEE Trans. Microw. Theory Tech., vol. 33, no. 10, pp. 960-966, Oct. 1985.

[16] R.-B. Wu and J.-C. Yang, "Boundary integral equation formulation of skin effect problems in multiconductor transmission lines," IEEE Trans. Magn., vol. 25, no. 4, pp. 3013-3015, Jul. 1989.

[17] R. Faraji-Dana and Y. Chow, "The current distribution and ac resistance of a microstrip structure," IEEE Trans. Microw. Theory Tech., vol. 38, no. 9, pp. 1268-1277, Sep. 1990.

[18] — "Ac resistance of two coupled strip conductors," Microwaves, Antennas and Propagation, IEE Proceedings $H$, vol. 138, no. 1, pp 37-45, Feb. 1991.

[19] M. Tsuk and J. Kong, "A hybrid method for the calculation of the resistance and inductance of transmission lines with arbitrary cross sections," IEEE Trans. Microw. Theory Tech., vol. 39, no. 8, pp. 13381347, Aug. 1991

[20] G. Plaza, R. Marques, and F. Medina, "Quasi-TM MoL/MoM approach for computing the transmission-line parameters of lossy lines," IEEE Trans. Microw. Theory Tech., vol. 54, no. 1, pp. 198-209, Jan. 2006.

[21] G. Antonini, A. Orlandi, and C. Paul, "Internal impedance of conductors of rectangular cross section," IEEE Trans. Microw. Theory Tech., vol. 47, no. 7, pp. 979-985, Jul. 1999.

[22] F. Medina, R. Marques, G. Antonini, A. Orlandi, and C. Paul, "Comments on internal impedance of conductors of rectangular cross section [and author's reply]," IEEE Trans. Microw. Theory Tech., vol. 49, no. 8, pp. 1511-1513, Aug. 2001.

[23] W. Heinrich, "Comments on internal impedance of conductors of rectangular cross section," IEEE Trans. Microw. Theory Tech., vol. 49, no. 3, pp. 580-581, Mar. 2001.

[24] A. Maue, "Zur Formulierung eines allgemeinen Beugungs-Problems durch eine Integralgleichung," Zeitschrift für Physik A Hadrons and Nuclei, vol. 126, no. 7-9, pp. 601-618, Jul. 1949.

[25] J. Meixner, "The behavior of electromagnetic fields at edges," IEEE Trans. Antennas Propag., vol. 20, no. 4, pp. 442-446, Jul. 1972.

[26] J. Van Bladel, Singular Electromagnetic Fields and Sources. New York: Oxford University Press, 1991.

[27] J. Geisel, K.-H. Muth, and W. Heinrich, "The behavior of the electromagnetic field at edges of media with finite conductivity," IEEE Trans. Microw. Theory Tech., vol. 40, no. 1, pp. 158-161, Jan 1992.

[28] D. De Zutter and L. Knockaert, "Skin effect modeling based on a differential surface admittance operator," IEEE Trans. Microw. Theory Tech., vol. 53, no. 8, pp. 2526-2538, Aug. 2005.

[29] T. Demeester and D. De Zutter, "Quasi-TM transmission line parameters of coupled lossy lines based on the Dirichlet to Neumann boundary operator," IEEE Trans. Microw. Theory Tech., vol. 56, no. 7, pp. 16491660, Jul. 2008.

[30] _ "Construction of the Dirichlet to Neumann boundary operator for triangles and applications in the analysis of polygonal conductors," IEEE Trans. Microw. Theory Tech., vol. 58, no. 1, pp. 116-127, Jan. 2010.

[31] _ - "Modeling the broadband resistive and inductive behavior of polygonal conductors," in International Conference on Electromagnetics in Advanced Applications, Torino, Italy, Torino, Italy, Sep. 2009.

[32] A. Omar and K. Schunemann, "Application of the generalized spectraldomain technique to the analysis of rectangular waveguides with rectangular and circular metal inserts," IEEE Trans. Microw. Theory Tech., vol. 39, no. 6, pp. 944-952, Jun. 1991. 


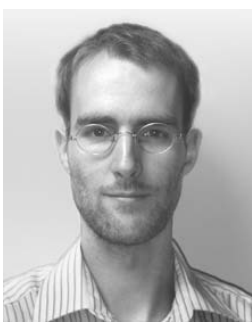

Thomas Demeester was born in 1982 in Ghent, Belgium. He received his M. Sc. Degree in electrical engineering from Ghent University in 2005, after a one-year period at ETH Zürich for his master thesis in the field of time-domain electromagnetics. $\mathrm{He}$ is currently working toward his Ph.D. degree at Ghent University, as a Research Fellow of the Fund for Scientific Research, Flanders. His research concerns electromagnetic field calculations in the presence of highly lossy media, and the development of transmission line models for interconnects.

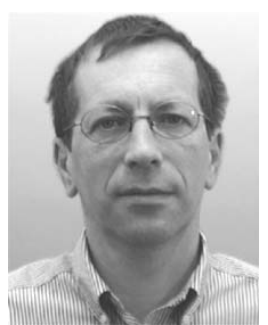

Daniël De Zutter was born in 1953. He received his M. Sc. Degree in electrical engineering from Ghent University in 1976. From 1976 to 1984 he was a research and teaching assistant at the same university. In 1981 he obtained a Ph. D. degree and in 1984 he completed a thesis leading to a degree equivalent to the French Aggrégation or the German Habilitation. From 1984 to 1996 he was with the National Fund for Scientific Research of Belgium. $\mathrm{He}$ is now a full professor of electromagnetics. Most of his earlier scientific work dealt with the electrodynamics of moving media. His research now focusses on all aspects of circuit and electromagnetic modelling of high-speed and high-frequency interconnections, packaging, on-chip interconnect and on numerical solutions of Maxwell's equations. As author or co-author he has contributed to more than 150 international journal papers. In 2000 he was elected to the grade of Fellow of the IEEE. In the past 4 yeas he served as dean of the Faculty of Engineering of Ghent University. He is an associate editor for the MTTTransactions. 